\title{
Relationship Between Vasospasm and Serum Chromogranin A Levels in an Animal Subarachnoid Hemorrhage Model
}

\author{
Halil Can KUCUKYILDIZ', Salih Kursat SIMSEK ${ }^{2}$, Almila SENAT ${ }^{3}$, Ahmet Gurhan GURCAY4, Ceylan BAL ${ }^{3}$, \\ Ercan $\mathrm{BAL}^{4}$ \\ ${ }^{1}$ Turkish Ministry of Health, Tokat State Hospital, Department of Neurosurgery, Tokat, Turkey \\ ${ }^{2}$ Turkish Ministry of Health, Ankara City Hospital, Department of Neurosurgery, Ankara, Turkey \\ ${ }^{3}$ Ankara Yildirim Beyazit University Faculty of Medicine, Department of Biochemistry, Ankara, Turkey \\ ${ }^{4}$ Ankara Yildirim Beyazit Faculty of Medicine, Department of Neurosurgery, Ankara, Turkey
}

This study has been presented as an oral presentation at the $32^{\text {th }}$ Scientific Congress of Turkish Neurosurgical Society between 20 and 24 April 2018 at Antalya, Turkey.

Corresponding author: Halil Can KUCUKYILDIZ drhalilcan@gmail.com

\section{ABSTRACT}

AIM: To analyze Chromogranin A levels on vasospasm in an experimental subarachnoid heamorrhage (SAH) model.

MATERIAL and METHODS: Sixteen Wistar Albino male rats were used in study. Two groups are formed; first was consisting of 8 rats that experimental SAH was performed on them, second group was control group that nothing was done. Animals were sacrified fourtyeight hours later subarachnoid heamorrhage was occured. Peripheral venous blood samples were taken from the experimental group before SAH formation, 15 minutes, 75 minutes after experimental SAH formation and 48 hours as peak of vasospasm. Simultaneous peripheral venous blood samples were also collected from the control group. Blood samples were biochemically evaluated after centrifugation and serum Chromogranin A levels were studied.

RESULTS: Serum chromogranin A levels increased statistically significant $(p<0.05)$ at the 15th minute after SAH, as the samples obtained from the experimental and control groups were anticipated as a result of the statistical analysis of the data after the biochemical examinations.

CONCLUSION: In all these findings, we concluded that Chromogranin A could be used as a marker for the investigation of endocrine stress in the early period of post-SAH vasospasm and it could be proved by more studies.

KEYWORDS: Subarachnoid haemorrhage, Vasospasm, Chromogranin A, Endocrin stress, Rat

\section{INTRODUCTION}

C erebral vasospasm is a pathological process that can occur after subarachnoid haemorrhage (SAH). It results from reversible constrictions in high-capacity intradural arteries at the base of the brain caused by blood, blood metabolites and other chemical substances. Cerebral vasospasm can develop slowly, and it exhibits a high degree of variation. It is usually associated with decreased perfusion and ischaemic neurological loss in the distal region of the affected artery. The oxidative stress in cerebral tissue after cerebral vasospasm is associated with cerebral ischaemia and reperfusion injury, one of the most important causes of mortality and morbidity following $\mathrm{SAH}$. Mechanisms underlying the etiopathogenesis of cerebral vasospasm have been the subject of recent studies $(18,21)$.

Chromograninsareacidic glycoproteinsfoundinneurosecretory granules (12). The chromogranin family includes chromogranin $\mathrm{A}(\mathrm{CgA}), \mathrm{B}$ and $\mathrm{C}$, and secretogranin III, IV, V and VI $(5,12)$.
Halil Can KUCUKYILDIZ (D) : 0000-0003-0922-1750

Salih Kursat SIMSEK (1D): 0000-0001-5000-9052

$\begin{array}{lll}\text { Almila SENAT } & \text { (1) : 0000-0002-5806-562X }\end{array}$
Ahmet Gurhan GURCAY (1) : 0000-0002-8810-938X

Ceylan BAL $\quad$ (1) : 0000-0002-1678-1281

Ercan BAL (1) : 0000-0003-0138-2090 
The studies have shown that CgA, an acidic glycoprotein involved in the regulation of secretory proteins, is common in all endocrine, neuroendocrine and secretory vesicles in the nervous system $(1,10,14)$, where it is found with peptide and amine hormones. It is also observed in dense core synaptic granules found in many areas of the gastrointestinal tract, as well as in the adeno- and neurohypophysis, the immune system, and the central nervous system's atrial myocardium. In a previous study, we analysed these differences analytically and found that $\mathrm{CgA}$ exhibits high antigenic heterogeneity (13). Because of its extensive expression in the neuroendocrine system, CgA has been used as a general biomarker for neuroendocrine cells, with high CgA levels observed in the presence of neuroendocrine tumours $(12,13,20)$.

The aim of this study, therefore, was to investigate the relationship between serum levels of $\mathrm{CgA}$, a catecholamine metabolite with vascular endothelial effects, and the occurrence of vasospasm in the anterior and posterior circulation following SAH.

\section{MATERIAL AND METHODS}

The study was approved by the Ethics Committee of Kobay DHL Co. (approval number 24503.08. 2017).

\section{Animal Model of SAH}

This study used healthy, adult, sixteen Wistar Albino male rats (mean weight, $150 \mathrm{~g}$ ) that had not been used in any previous studies. Two groups are formed; first was consisting of 8 rats that experimental SAH was performed on them, second group was control group that nothing was done. Animals were sacrified fourtyeight hours later subarachnoid heamorrhage was occured. The animals were randomly divided into control and SAH groups. They were anaesthetised by the intramuscular injection of $50 \mathrm{mg} / \mathrm{kg}$ of a $10 \%$ ketamine/2\% xylazine mixture, and the SAH group rats additionally received an injection of $0.2 \mathrm{~mL}$ of autologous venous blood into the cisterna magna after removing the same amount of cerebrospinal fluid (CSF) via a lumbar puncture. After $48 \mathrm{~h}$, the time the most severe vasospasm was expected to occur, the animals were sacrificed, decapitated, and the brain and brainstem carefully removed in toto to avoid damage.

\section{Blood Sampling}

Ten minutes before administering the anaesthetic, $0.2 \mathrm{~mL}$ of peripheral venous blood was drawn from each rat via the orbital sinus, as described previously (15). At 15 and 75 minutes after the induction of SAH in the experimental group, and immediately prior to sacrifice at 48 hours, 0.2 $\mathrm{mL}$ of venous blood was taken from animals in both groups (19). After centrifugation at 5,000 rpm, the blood samples were stored at $+4{ }^{\circ} \mathrm{C}$. $\mathrm{CgA}$ levels were measured using the Hangzhou Eastbiopharm ELISA kit with a KHB ST-360 ELISA Reader.

\section{Statistical Analysis}

The statistical analysis for the study was performed using IBM SPSS Statistics v 23.0 (IBM Corp., Armonk, NY, USA). The difference between groups before and after the induction of SAH was compared using the Friedman and Wilcoxon tests. In the results, $p$-values less than 0.005 were considered statistically significant.

\section{RESULTS}

The blood collected 15 min after the induction of SAH in the experimental group showed significantly higher levels of serum $\mathrm{CgA}$ in the experimental group compared with the control group $(p=0.012)$. There were no significant differences in CgA levels between the groups for the blood samples collected at baseline (10 min before the induction of SAH), $75 \mathrm{~min}$, and $48 \mathrm{~h}$ (Table I, Figure 1). Also, microscopic view of focal edema and neurolysis areas in a preparate obtained from the frontal cortex of a SAH induced subject and stained with Hematoxylin Eosin is shown Figure 2.

\section{DISCUSSION}

$\mathrm{SAH}$-related cerebral vasospasm has been known for more than half a century; nevertheless, it remains a complex, multifactorial problem for patients with ruptured intracranial aneurysms. Recently, there have been considerable improvements in the treatment for intracranial aneurysm via surgical and endovascular approaches. However, despite numerous studies, the pathogenesis of SAH-related vasospasm remains obscure, and the most appropriate treatment has not been fully established. It has been understood since the first half of the 1900s that changes occur in the cerebral vessel wall after $\mathrm{SAH}$. Much more conservative, prophylactic treatment has gained importance than the treatment of most of the changes up to date. Establishing the mechanisms of vasospasm development and identifying appropriate treatment methods could therefore revolutionise vascular neurosurgery.

Ideally, studies on the pathogenesis and treatment of cerebral vasospasm would be performed using human cerebral arteries. However, human vessels cannot be used for in vivo experiments and the information available from examining postmortem vasospastic human arteries is limited, so various animal models have been developed. In vitro studies of $\mathrm{SAH}$ and vasospasm involve the removal of the artery to be examined immediately after the animal's death; however, a limitation of these studies is that the arteries are separated from their natural environment, so the information that can be obtained from them will be limited. For this reason, in vivo models for SAH and vasospasm have been developed. In an in vivo model, at least one living animal should have blood elements surrounding the vessel. These blood demolition elements will cause vasoconstriction over time. In 1992, Kassell et al. created an experimental model of SAH by feeding autologous blood into the cisterna magna of rabbits and examining morphological changes of the artefacts periodically over 6 days. Although none of the animals developed a neurological deficit, the animals sacrificed after 2 days exhibited a thick coagulum around the basilar artery; in the animals sacrificed over the following days, this coagulant was graded gradually (9). 
Table I: Serum Chromogranin A Levels in Rats with Induced SAH ( $\mathrm{n}=16)$ at Baseline $(-10 \mathrm{~min})$ and at 15 and 75 min and $48 \mathrm{~h}$ After Inducing the SAH

\begin{tabular}{|c|c|c|c|c|c|}
\hline & Minutes after SAH & Mean \pm SD & Median & Range & $\mathbf{p}^{\mathbf{a}}$ \\
\hline \multirow[t]{4}{*}{ Serum chromogranin A, ng/mL } & -10 & $78.56 \pm 21.52$ & 86.18 & 66.15 & \\
\hline & +15 & $141.09 \pm 48.24$ & 130.62 & 143.25 & $0.012^{\star}$ \\
\hline & +75 & $98.60 \pm 39.24$ & 85.62 & 115.17 & 0.767 \\
\hline & +2880 & $81.85 \pm 15.84$ & 76 & 50.99 & 0.953 \\
\hline
\end{tabular}

a Wilcoxon matched pairs test, compared with the control group levels. SAH: subarachnoid hemorrhage.

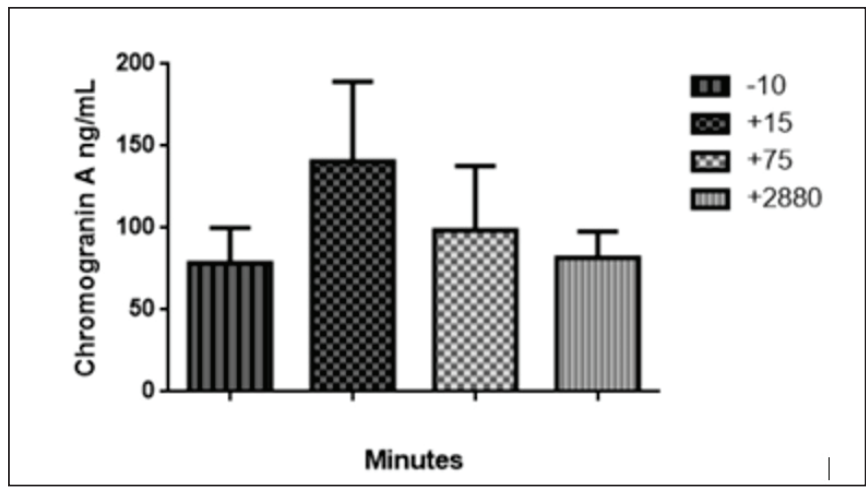

Figure 1: Mean $( \pm S D)$ chromogranin A levels in rats with induced subarachnoid haemorrhage $(\mathrm{SAH})$ at baseline $(-10 \mathrm{~min})$ and at 15 and $75 \mathrm{~min}$ and $48 \mathrm{~h}$ after inducing the $\mathrm{SAH}$.

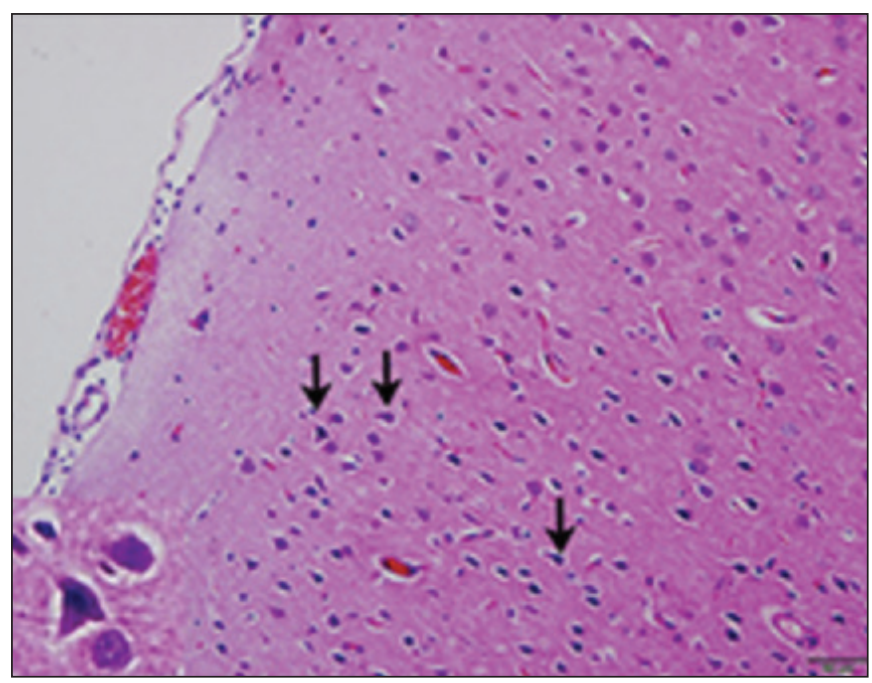

Figure 2: Frontal cortex of a rat model of subarachnoid haemorrhage showing the focal edema and neurolysis, which are indicated by arrows (Staining Method - Haematoxylin and Eosin; Light Microscope; magnification, 10x).

When a patient suffers $\mathrm{SAH}$, it is important to assess whether he or she is likely to experience vasospasm, because this affects the timing of post-bleeding treatment and the aggressiveness of the treatment, as well as the need for vasospasm treatment and the patient's length of hospital stay. Thus, it could be valuable to determine whether there is an association between the endocrine stress response and vasospasm after bleeding. It has been proposed that, after $\mathrm{SAH}$, catecholamine and its metabolites may stimulate vasospasm; thus, levels of these may provide an indicator for the development of vasospasm, especially in the early period.

We chose to use rats in our study because they are easy to find, cheap, easy to maintain and exhibit vasospasm comparable to that in humans. Acute vasospasm in rats reaches a maximum at $48 \mathrm{~h}$ after the induction of SAH (8). Experimental SAH has been induced via three basic approaches, with many methods developed based on these techniques. The first approach is for blood to be collected around the artery. The second is surgical dissection of the artery, with the placement of autologous blood from another artery around it $(6,17)$. Finally, the most common technique is the injection of autologous arterial blood into the subarachnoid space $(15,16)$. This method can be applied as single, double or triple bleeding. In rat models of vasospasm, the rats typically do not develop neurological deficits, but instead show intense collateral circulation (16). In addition, as in humans, the arterial system in the subarachnoid space does not develop adventitia, vasovasorums are absent and the arteries are fed from the CSF. In rats, there is a more extensive choice of the arteries for morphometric measurements in the posterior circulation. Studies have shown that vasospasm in rats follows a biphasic pattern. The resulting acute spasm should be limited to a certain timeframe. Notably, the late-onset vasospasm seen on the second day in rats develops earlier than it does in other species. Vasospasm seen in humans peaks on the seventh day, the time when neurological findings are observed clinically.

Because of the morphological similarity of cerebral vascular tissues, in both humans and rats, an increase in intracranial pressure occurs after bleeding; consequently, there is a decrease in cerebral perfusion pressure. As a result, there is closure of the rete vasorum (which allows the cerebral vascular tissues to feed from the CSF) because it does not contain adventitia with the pores of the entire vessel layer with blood clots. These clogged constructions cannot function properly and so ischaemia results in vascular and neuronal tissues. The destruction of the vascular cell membrane is a complex event, with subsequent reactions including acute inflammation, the production of free radicals, lipid peroxidation, damage to vascular endothelial cells, a decrease in nitric oxide levels, an increase in endothelin content, and the accumulation of intracellular calcium. 
These reactions have been used to explain vasospasm. The most popular hypothesis is that the vasospasm develops with the destruction of blood elements; according to this, erythrocytes and platelet-destroying spasmogens are activated after the onset of $\mathrm{SAH}$, triggering vasospasm. These plasmids include serotonin, prostaglandins, catecholamines, angiotensin and oxyhemoglobin (7). However, it has been show in laboratory experiments that none of these can induce (or resolve) vasospasm independently (7). The onset of hemolysis immediately after $\mathrm{SAH}$ and the continuing lysis and phagocytosis of erythrocytes have attracted the attention of researchers, with studies showing that oxyhemoglobin is the main factor responsible for vasospasm (16). When oxyhemoglobin binds nitric oxide, levels of cyclic guanosine monophosphate (cGMP) decrease and vasospasm develops (7). Free iron with oxyhemoglobin increases lipid peroxidation; as a result, there is an increase in free radical production. Free radicals may then disrupt the blood-brain barrier, resulting in endothelial damage (16). The increase in iron in the hemolysed environment after $\mathrm{SAH}$ suggests that free radicals may be responsible for cerebral vasospasm and neuronal damage (11). Oxyhemoglobin catalyses the formation of oxygen free radicals by increasing lipid peroxidation activity in conjunction with the free iron. This results in endothelial damage and an increase in intracellular calcium. Calcium and calmodulin, a calcium-dependent enzyme, play an important role in vascular smooth muscle regulation (11).

The first identified effect of antioxidants was the protection of membrane lipids against peroxidation. Antioxidants were therefore defined as molecules that inhibit lipid peroxidation. The definition of antioxidants has since been expanded to include molecules that exert protective effects against the oxidation of other target molecules, such as proteins, nucleic, acids and carbohydrates.

Cerebral vasospasm, which is observed soon after the subarachnoid autologous blood injection in experimental animals, is extremely rare in humans during the first three days after $\mathrm{SAH}$. Vasospasm typically begins on day 3 , reaching its maximal level during days 6-10 $(2,3)$. Cerebral vasospasm is associated with a high degree of mortality and morbidity. It has been suggested that it may be possible to predict the occurrence of cerebral vasospasm by measuring the levels of various blood markers in the early period after SAH formation. This approach was examined by Espiner et al. and Nyberg et al., who investigated the neuro-cardio-endocrine response and early endocrine stress response to $\mathrm{SAH}$; however, they found no indicator of cerebral vasospasm in their day-to-day studies $(4,14)$. This shows the need for an extensive, comprehensive study of potential markers for cerebral vasospasm that may develop after $\mathrm{SAH}$, especially in the early period. This is especially important in locations without a vasospasm clinic.

In a study by Nyberg et al., experimentally induced SAH resulted in elevated serum levels of adrenocorticotrophic hormone and cortisol, increased plasma catecholamines, and the increased excretion of vanillylmandelic acid derivatives during the vasospasm (14). Catecholamines and their metabolite CgA showed a significant increase during the early period of vasospasm (15 min). During the progressive phase of vasospasm, there is a gradual increase in the excretion of catecholamine metabolites in the urine, associated with the shortened plasma half-lives of catecholamines. Thus, it is unlikely that catecholamines and chromogranins present in the constellations will increase in the late phase of stress after $\mathrm{SAH}$, or even during the period when vasospasms are expected to reach a peak in animal models (48 h). Nevertheless, they are thought to play a role in the post-stress acute endocrine response, and the increase in catecholamines and chromogranins in the blood during the early period of vasospasm offers the potential that they could be used as predictors of vasospasm before any clinical findings are observed.

In our study, we used rats differently from Nyberg et al. Also, measurement and sampling period is expanded up to 48 hours, which is the time that considered to vasospasm has peaked. Eventually, any statistically significant increasing detected in $\mathrm{CgA}$ levels at $48 \mathrm{~h}$ after $\mathrm{SAH}$. But, $\mathrm{CgA}$ showed a significant increase during the early period of vasospasm (15 min) similarly with the study published hitherto. Consequently, the high levels of serum $\mathrm{CgA}$ in the early period after $\mathrm{SAH}$ in both studies conducted in two different animal groups are valuable in terms of being a marker in the early period of vasospasm.

The aim of the present study was to investigate the relationship between cerebral vasospasm and post-SAH stress levels of $\mathrm{CgA}$, a neuroendocrine marker known to play a role in vascular system regulation. We measured $\mathrm{CgA}$ levels in plasma samples obtained at various time points from rats with and without SAH. This showed that there was a significant increase in $\mathrm{CgA}$ in the early period (15 min) after $\mathrm{SAH}$, but not at any of the other time points, which showed a decreasing trend. These findings were consistent with those of Nyberg et al., who assessed the early endocrine stress response after $\mathrm{SAH}$ and reported high levels of $\mathrm{CgA}$ in the early post-SAH period at the same time (15 min) as in our study (14). These results suggest that the measurement of plasma levels of $\mathrm{CgA}$ early in the post-SAH period may provide a marker for vasospasm.

\section{CONCLUSION}

$\mathrm{CgA}$ levels can be measured as part of the investigation of the development of the endocrine stress response against cerebral vasospasm after SAH. However, only a limited number of studies of this have been conducted. These have shown similar increases in $\mathrm{CgA}$ levels associated with the early stage of SAH and the vasospasm endocrine stress response. It is possible that $\mathrm{CgA}$ can be used as a marker in cerebral vasospasm; however, there is a need for more extensive work in this area.

\section{REFERENCES}

1. Banks $P$, Helle $K$ : The release of protein from the stimulated adrenal medulla. Biochem J 97:40C-41C, 1965 
2. Barry KJ, Scott RM: Effect of intravenous ethanol on cerebral vasospasm produced by subarachnoid blood. Stroke 10:535537,1979

3. Echlin FA: Current concepts in the etiology and treatment of vasospasm. Clin Neurosurg 15:133-160, 1968

4. Espiner EA, Leikis R, Ferch RD, MacFarlane MR, Bonkowski JA, Frampton CM, Richards AM: The neuro-cardio-endocrine response to acute subarachnoid haemorrhage. Clin Endocrinol (Oxf) 56:629-635, 2002

5. Feldman SA, Eiden LE: The chromogranins: Their roles in secretion from neuroendocrine cells and as markers for neuroendocrine neoplasia. Endocr Pathol 14:3-23, 2003

6. Findlay JM, Weir BK, Kanamaru K, Espinosa F: Arterial wall changes in cerebral vasospasm. Neurosurgery 25:736-745, discussion 745-736, 1989

7. Harrod CG, Bendok BR, Batjer HH: Prediction of cerebral vasospasm in patients presenting with aneurysmal subarachnoid hemorrhage: A review. Neurosurgery 56:633654, discussion 633-654, 2005

8. Huang Q, Wang G, Hu YL, Liu JX, Yang J, Wang S, Zhang HB: Study on the expression and mechanism of inflammatory factors in the brain of rats with cerebral vasospasm. Eur Rev Med Pharmaco 21:2887-2894, 2017

9. Kassell NF, Helm G, Simmons N, Phillips CD, Cail WS: Treatment of cerebral vasospasm with intra-arterial papaverine. $J$ Neurosurg 77:848-852, 1992

10. Kim H, Britton GL, Peng T, Holland CK, McPherson DD, Huang SL: Nitric oxide-loaded echogenic liposomes for treatment of vasospasm following subarachnoid hemorrhage. Int J Nanomedicine 9:155-165, 2014

11. Liu-Deryke $\mathrm{X}$, Rhoney $\mathrm{DH}$ : Cerebral vasospasm after aneurysmal subarachnoid hemorrhage: An overview of pharmacologic management. Pharmacotherapy 26:182-203, 2006

12. Louthan O: Chromogranin a in physiology and oncology. Folia Biol (Praha) 57:173-181, 2011
13. Metz-Boutigue MH, Garcia-Sablone $\mathrm{P}$, Hogue-Angeletti $\mathrm{R}$, Aunis D: Intracellular and extracellular processing of chromogranin A. Determination of cleavage sites. Eur J Biochem 217:247-257, 1993

14. Nyberg C, Karlsson T, Hillered L, Stridsberg M, Ronne Engstrom $\mathrm{E}$ : The early endocrine stress response in experimental subarachnoid hemorrhage. PLoS One 11:e0151457, 2016

15. Parasuraman S, Raveendran R, Kesavan R: Blood sample collection in small laboratory animals. J Pharmacol Pharmacother 1:87-93, 2010

16. Pluta RM, Afshar JK, Boock RJ, Oldfield EH: Temporal changes in perivascular concentrations of oxyhemoglobin, deoxyhemoglobin, and methemoglobin after subarachnoid hemorrhage. J Neurosurg 88:557-561, 1998

17. Prunell GF, Mathiesen T, Diemer NH, Svendgaard NA: Experimental subarachnoid hemorrhage: Subarachnoid blood volume, mortality rate, neuronal death, cerebral blood flow, and perfusion pressure in three different rat models. Neurosurgery 52:165-175, discussion 175-176, 2003

18. Santiago-Sim T, Fang X, Hennessy ML, Nalbach SV, DePalma SR, Lee MS, Greenway SC, McDonough B, Hergenroeder GW, Patek KJ, Colosimo SM: THSD1 (Thrombospondin Type 1 Domain Containing Protein 1) mutation in the pathogenesis of intracranial aneurysm and subarachnoid hemorrhage. Stroke 47:3005-3013, 2016

19. Schurkamper M, Medele R, Zausinger S, Schmid-Elsaesser R, Steiger HJ: Dexamethasone in the treatment of subarachnoid hemorrhage revisited: A comparative analysis of the effect of the total dose on complications and outcome. J Clin Neurosci 11:20-24, 2004

20. Tota B, Quintieri AM, Di Felice V, Cerra MC: New biological aspects of chromogranin A-derived peptides: Focus on vasostatins. Comp Biochem Physiol A Mol Integr Physiol 147:11-18, 2007

21. van Donkelaar CE, Bakker NA, Veeger NJM, Uyttenboogaart M, Metzemaekers JDM, Luijckx GJ, Groen RJM, van Dijk JMC: Predictive factors for rebleeding after aneurysmal subarachnoid hemorrhage: Rebleeding aneurysmal subarachnoid hemorrhage study. Stroke 46:2100-2106, 2015 